\title{
Current problems associated with the microbiological point-of-care testing of respiratory tract infections in primary care
}

\author{
“...healthcare providers may need to consider giving incentives to general \\ practitioners if sufficient numbers are to adopt and utilize these 'first-generation' \\ devices. ”
}

\author{
Wendy E Kaman', Gijs Elshout ${ }^{2}$, Patrick JE Bindels ${ }^{2}$, Konstantinos Mitsakakis ${ }^{3,4}$ \\ \& John P Hays*,1
}

First draft submitted: 12 December 2015; Accepted for publication: 25 February 2016;

Published online: 11 May 2016

Currently, the world is facing a potential 'antibiotic apocalypse', whereby clinically relevant bacteria are becoming increasingly resistant to all currently available antibiotics [1]. Several factors are contributing to this global increase in antibiotic resistance, including the unnecessary prescription of antibiotics for nonbacterial (viral and fungal) infections [2]. General practitioners (GPs) sit on the front line of antibiotic prescribing practice, having regular and broad-based contact with members of society that present to their offices with a range of disease states. One of the major reasons for visiting a GP is when a patient is apparently suffering from a respiratory tract infection [3]. The accurate diagnosis of the cause (i.e., the causative pathogen) of this infection can be difficult for the GP to make when only based on clinical symptoms. In the absence of sufficient diagnostic or clinical evidence, the GP may decide to prescribe antibiotics, or alternatively may withhold antibiotic therapy (suspecting a viral infection) when antibiotic therapy is actually required. On the one hand, the 'overprescribing' of antibiotics for nonbacterial infections may not only lead to the acquisition of antibiotic resistance in the resident flora of the patient, but also result in unnecessary healthcare costs for the patient and society [4]. It will also expose the patients to unwanted side effects from the antibiotic [5]. On the other hand, not prescribing antibiotics in case of a bacterial pneumonia, may result in extra morbidity for the patient and subsequent healthcare costs [6].

Access to point-of-care (POC) tests may reduce the prescription of antibiotics in primary care [7], although current POC testing performed by GPs for diagnosing respiratory tract infections tends to rely on the use of nonspecific biomarkers, such as CRP [8]. These biomarkers only provide information on the likelihood of a complicated (lower) respiratory tract infection being present. They do not identify pathogens or any antibiotic resistance that may be associated with a pathogen. For this reason, more advanced POC testing devices are currently being developed for specific use in the primary care environment. Such devices could potentially accurately diagnose

'Department of Medical Microbiology \& Infectious Diseases, Erasmus University Medical Center Rotterdam (Erasmus MC)

Wytemaweg 80, 3015 CN, Rotterdam, The Netherlands

2Department of General Practice, Erasmus University Medical Center Rotterdam (Erasmus MC), Wytemaweg 80, 3015 CN, Rotterdam,

The Netherlands

${ }^{3}$ Hahn-Schickard, Georges-Koehler-Allee 103, 79110 Freiburg, Germany

${ }^{4}$ Laboratory for MEMS Applications, IMTEK - Department of Microsystems Engineering, University of Freiburg,

Georges-Koehler-Allee 103, 79110 Freiburg, Germany

*Author for correspondence: j.hays@erasmusmc.nl

Future

\section{KEYWORDS}

- antibiotic resistance

- point-of-care testing

- primary care $\bullet$ respiratory

tract infections 


\section{"If a (commercially) successful infectious disease point-of-care diagnostic device is to be developed for primary care, the choice of target pathogens (and/or genes) will be crucial.”}

the causative pathogen associated with a respiratory tract infection and any accompanying antibiotic resistance genes (if present), thereby potentially helping the GP to personalize their antibiotic prescribing practices to the needs of each patient.

Unfortunately, however, many hurdles currently exist to the successful implementation of infectious disease POC diagnostics into primary care, the most important of which are described below.

\section{Specimen sampling \& processing}

In general practice, sample collection for respiratory tract infections is generally limited to the collection of swabs, aspirates or sputum (for lower respiratory tract infections). For the detection of biomarkers, blood samples may also be taken. Nose, throat or nasopharyngeal aspirates could potentially be taken by GPs, although nasopharyngeal aspirates tend not to be well tolerated by patients as it is an uncomfortable sampling procedure. Throat or nose swabs can be collected, but may provide very little information about the actual pathogen associated with a lower respiratory infection, where a sputum sample would be preferred [9]. However, it may be difficult to obtain a sputum sample during the patient's visit, especially from pediatric patients (the majority of patients with fever and cough visiting the GP). Also, the value of taking a nose or throat swab for determining the cause of otitis media infections is debatable. Finally, the type and complexity of sample preparation may be dependent on the type of specimen taken. For example, is it possible to develop simple protocols that can adequately process all of these different samples within a POC diagnostic device? Are manual steps required prior to sample analysis, is specific training of these steps required and how well accepted are these steps by the end users? The answer to these questions may have a large impact on the development and final usefulness of infectious disease POC diagnostic devices.

\section{Choice of targets}

If a (commercially) successful infectious disease POC diagnostic device is to be developed for primary care, the choice of target pathogens (and/or genes) will be crucial. However, deciding on which targets are most suitable for inclusion is very difficult. Although long lists of respiratory viral and bacterial pathogens and the accompanying antibiotic resistance are available, the prevalence of these pathogens and genes within different countries of the world - and indeed within Europe itself - varies greatly [10], not only with respect to geography, but also with respect to time [11]. For example, the incidence of viral respiratory tract infections, such as influenza and respiratory syncytial virus, may not only show seasonal variation, but also annual variation $[12,13]$. Will the sales of an influenza and/or respiratory syncytial virus POC test during years of low incidence be sufficient to maintain commercial production? Further, will companies be able to scale-up their production when peak years of infection occur? Let us also consider a POC device that can detect many different viruses and/or bacteria associated with respiratory tract infections. Will the diagnosis of, for example, 'human bocavirus' or 'human metapneumovirus' provide any useful information to the GP? Will the GP know what these viruses are? Should the GP prescribe antibiotics if both a virus and bacterial pathogen are detected by a POC device? This decision will probably depend upon which virus and which bacterium is detected but, even so, the role of individual viruses and bacteria in respiratory tract infections can be unclear. For example, children may be carriers of potentially pathogenic bacteria, including Streptococcus pneumoniae, Streptococcus pyogenes and Haemophilus influenza. Even though these potential pathogens can be cultured from such 'carriers', carriers can remain perfectly healthy (although this situation may change as they become adults [14]). How does a GP determine whether a positive result for any of these potentially pathogenic bacteria are the actual cause of a respiratory tract infection in children? With respect to mixed infections, what does the GP prescribe for a child if, for example, both 'human coronavirus 229E' and S. pneumoniae are detected? Perhaps in this case, biomarkers such as CRP could play an additional role in guiding the decision of the GP? Does this then mean that these biomarkers have to be included in all respiratory tract infection POC devices, or at least be available for supplemental testing if required? Perhaps biomarkers would not have to be used if instead we quantified the level of potential bacterial pathogens in the patient? Unfortunately, there is no worldwide or 
European consensus on the 'standard' level of carriage of potential bacterial pathogens, such as $S$. pneumoniae, S. pyogenes and H. influenza, in healthy children compared with sick children. This lack of data seriously compromises the use of quantification assays for distinguishing between levels associated with healthy carriage and disease, especially in children. However, this does not mean to say that quantification per se does not have its advantages. For example, a hospital-based physician may be interested in the quantification of antibiotic and antiviral resistance genes/gene mutations during therapy for life-threatening diseases. However, this application may only be useful in specific clinical settings.

\section{Advice for the physician}

Based on the problems described above, perhaps a POC diagnostic device should include software, or instructional material, that guides the decision-making of a physician based on the results of the test performed in addition to clinical signs and symptoms. This advice could include: which organism most likely significantly contributes to the current infection - may be useful for mixed infections; which antibiotics (if any) should be prescribed and what further tests should be performed in order to confirm the diagnosis? In this way, the physician will be supported in his/her clinical decision making as to whether an antibiotic needs to be prescribed for patients presenting with symptoms of a respiratory tract infection. However, the problem here is that that type of antibiotic prescribed per general practice, or per country (if national guidelines exist) may vary at any given moment in time. Also, antibiotic resistance is not a static phenomenon and the continuing global increase in antibiotic resistance means that advice given by POC devices would have to be regularly updated. Further, the extra effort of continually monitoring national guidelines over time would be an extra financial burden on POC manufacturing companies. With respect to the most significant organism, this is not always obvious, and even a relatively simple POC device that could detect three viruses, three bacteria and three antibiotic resistance genes would require advice for 36 potential combinations, even if only two different variables, for example, a bacterium and a virus, were detected. Finally, if the POC device is to recommend additional confirmatory testing, then the GP may ask him/herself what the extra value is in investing in a POC device that requires additional testing.

In conclusion, developing an infectious disease POC diagnostic device for implementation in general practice is extremely difficult due to the lack of data and the fact that each country faces its own particular set of respiratory tract pathogens and antibiotic resistances, all of which (to add complexity to situation) may vary over time. However, the development of POC devices utilizing a range of modular components may allow global GPs to adapt their testing practices to meet the challenges of their own geographical location. Certainly, much more GP-specific data are required, including feedback regarding the needs of GPs as potential POC end users, and a common set of standards needs to be urgently developed if infectious disease POC diagnostic devices are to be successfully implemented into primary care. Currently, we are facing a 'chickenor-the -egg' situation whereby 'first-generation', but comprehensive, infectious disease POC diagnostic devices need to be accepted into primary care, so that accurate data and statistics can be collected in order to develop more accurate and cheaper 'second-generation' diagnostic devices that have clinical relevance to GPs, and ultimately to patients themselves. However, healthcare providers may need to consider giving incentives to GPs if sufficient numbers are to adopt and utilize these 'first-generation' devices. Several questions, therefore, remain: which targets are most informative for the treatment of respiratory tract infections in primary care? Who will fund the development of these 'first generation' devices? And, ultimately, who will pay for the introduction of these 'first generation' devices into general practice?

Financial and competing interests disclosure

This publication was supported by a Horizon 2020

European Union grant under grant agreement GA-633780 ("DIAGORAS"-www.diagoras.eu). The authors have no other relevant affiliations or financial involvement with any organization or entity with a financial interest in or financial conflict with the subject matter or materials discussed in the manuscript apart from those disclosed.

No writing assistance was utilized in the production of this manuscript.

\section{Open access}

This work is licensed under the AttributionNonCommercial-NoDerivatives 4.0 Unported License. To view a copy of this license, visit http://creativecommons.org/ licenses/by-nc-nd/4.0/ 


\section{References}

1 World Health Organization. Antimicrobial resistance: global report on surveillance (2014).

www.who.int/drugresistance/documents/

2 Gaynes R, Levy S. Improving outpatient antibiotic prescribing for respiratory tract infections: results of new algorithms used in European trials. Infect. Cont. Hosp. Ep. 36(6), 725-729 (2015).

3 NHS. Conditions: respiratory tract infections.

www.nhs.uk/conditions/Respiratory-tract

4 Guidos RJ. Combating antimicrobial resistance: policy recommendations to save lives. Clin. Infect. Dis. 52(5), S397-S428 (2011).

5 Get smart: know when antibiotics work. www.cdc.gov/getsmart/community/

6 Meterskya ML, Masterton RG, Lodec H, File TM Jr, Babinchake T. Epidemiology, microbiology, and treatment considerations for bacterial pneumonia complicating influenza. Int. J. Inf. Dis. 16(5), e321-e331 (2012).

7 Llor C, Bjerrum L, Munck A, Cots JM, Henández S, Moragas A. Access to point-ofcare tests reduces the prescription of antibiotics among antibiotic-requesting subjects with respiratory tract infections. Resp. Care 59(12), 1918-1923 (2014).

8 Biomarkers as point-of-care tests to guide prescription of antibiotics in patients with acute respiratory infections in primary care (review).

http://gaps.uq.edu.au/filething

9 Jeong JH, Kim KH, Jeong SH, Park JW, Lee SM, Seo YH. Comparison of sputum and nasopharyngeal swabs for detection of respiratory viruses. J. Med. Virol. 86(12), 2122-2127. (2014).

10 Bell BG, Schellevis F, Stobberingh E, Goossens H, Pringle M. A systematic review and meta-analysis of the effects of antibiotic consumption on antibiotic resistance. $B M C$ Infect. Dis. 14(13), 1-25 (2014).
11 Lagacé-Wiens PRS, Adam HJ, Low DE et al. Trends in antibiotic resistance over time among pathogens from Canadian hospitals: results of the CANWARD study 2007-2011. J. Antimicrob. Chemoth. 68(1), i23-i29 (2013).

12 Fowlkes A, Steffens A, Temte J et al. Influenza incidence surveillance project working group. Lancet Respir. Med. 3(9), 709-18. (2015).

13 Sirimi N, Miligkos M, Koutouzi F, Petridou E, Siahanidou T, Michos A. Respiratory syncytial virus activity and climate parameters during a 12-year period. J. Med. Virol. doi:10.1002/jmv.24430 (2015) (Epub ahead of print).

14 Adegbola RA, De Antonio R, Hill PC et al. Carriage of Streptococcus pneumoniae and other respiratory bacterial pathogens in low and lower-middle income countries: a systematic review and meta-analysis. PLoS ONE 9(8), e103293 (2014). 